\title{
¿Es posible hacer evidentes los procesos de metacognición en la resolución de problemas, fase $2^{1}$ ? $^{1}$
}

\author{
Is it possible to clear the processes of metacognition \\ in problem solving phase? \\ É possível limpar os processos de metacognição \\ em fase de resolução de problema?
}

Recibido: mayo de 2013

Aceptado: agosto de 2013
Daniel Alejandro Santos Ballén ${ }^{2}$

Gustavo Adolfo Lozada Cuervo ${ }^{3}$

\section{Resumen}

Segunda parte y continuación de la ponencia titulada ¿Es posible hacer evidentes los procesos de metacognición en la resolución de problemas? Extendiendo el análisis metacognitivo en la resolución de problemas hasta las fases de ataque y revisión ${ }^{4}$, Intentando hacer evidentes distintas acciones metacognitivas luego de un primer acercamiento al problema, hasta la construcción de una solución.

Palabras clave: Resolución de problemas; resolución y estrategias; metacognición; análisis metacognitivo; teorías de aprendizaje; constructivismo.

\begin{abstract}
Second part and continuation of the paper titled Is it possible to clear the processes of metacognition in problem solving? Extending the analysis metacognitive problem solving phases to attack and review1, Trying to clear various metacognitive actions after a first approach to the problem, to the construction of a solution.
\end{abstract}

Keywords: Troubleshooting, resolution and strategies, metacognition metacognitive analysis, learning theories, constructivism.

\section{Resumo}

Segunda parte e continuação do estudo intitulado É possível limpar os processos de metacognição na resolução de problemas? Estendendo o problema metacognitive análise resolver fases de ataque e review1, Tentar

1 Fase 1 expuesta en el 13 Encuentro Colombiano de Matemática Educativa bajo el nombre de: ¿Es posible hacer eviden tes los procesos de metacognición en la resolución de problemas?

2 Universidad Distrital Francisco José de Caldas, Maestría en Educación, Bogotá, Colombia. Contacto: das1032@gmail.com

3 Universidad Distrital Francisco José de Caldas, Maestría en Educación, Bogotá, Colombia. Contacto: gustavio90@hotmail.com

4 Mason, Burton y Stacey (1989) Pensar matemáticamente. Madrid, España: Editorial Labor. 
remover várias acções metacognitive depois de uma primeira abordagem para o problema, para a construção de uma solução.

Palavras-chave: Solução de problemas, resolução e estratégias, a análise metacognitiva metacognição, teorias de aprendizagem, o construtivismo.

\section{Planteamiento del problema}

Desde la construcción profesional como licenciados en Educación Básica con Énfasis en Matemáticas y la práctica pedagogía, ha surgido distintos cuestionamientos entorno a los procesos de enseñanza y aprendizaje de las matemáticas que se han llevado a cabo por medio de la implementación del modelo constructivista propuesto por Ausubel (1983), el cual centra su atención en el estudiante, en sus estructuras y estrategias cognitivas. Modelo basado en la comprensión del conocimiento por medio de su construcción, la cual está mediada por el estudiante, su entorno y el mismo conocimiento, ubicándose a las acciones mentales y razonamientos en un primer plano de esta mediación, estas acciones son estudio de la metacognición entendida como:

...el grado de conciencia o conocimiento que uno tiene sobre sus formas de pensar (procesos, eventos cognitivos) los contenidos de los mismos (estructuras) y la habilidad para controlar estos procesos con el propósito de organizarlos, revisarlos y modificarlos en función de sus resultados (Flavell 1985, citado en Jiménez V. 2004).

Según lo anterior, es posible afirmar que la idea de realizar un estudio metacognitivo entorno aun proceso de aprendizaje, puede permitir hacer evidente las distintas estructuras y estrategias cognitivas que se realizan, lo que puede generar un posible análisis de éstas a partir del contexto donde surjan en la resolución de problemas, lo cual es de interés para nosotros como investigadores, luego que desde nuestra posición como educadores según Jorba y Santamaría (Citado por Jiménez, 2004) "sería poco imaginable que una persona dedicada a generar conocimiento científico pudiera llegar a desarrollarlo sin representarse adecuadamente qué está buscando". Por lo cual se le da sentido a la pregunta de investigación expresada en los siguientes términos: ¿Cuáles acciones metacognitivas se logran hacer evidente en la resolución de un problema por estudiantes para maestro? El centro de estudio es el proceso metacognitivo realizado por los investigadores.

\section{Marco de referencia conceptual}

Teniendo en cuenta los dos aspectos fundamentales de esta investigación: la resolución de problemas y la metacognición, es necesario establecer diferencias y relaciones entre metacognición, resolución de problemas y análisis metacognitivo. Por un lado, la metacognición y la resolución de problemas se pueden relacionar a partir de tres dimensiones mencionadas por Ríos (citado en Jiménez, 2004) y las fases (abordaje, ataque y revisión), y procesos mencionadas por Mason, Burton y Stace (1989). Tales dimensiones son: planificar, monitorizar y evaluar; éstas se muestran como vitales para las pretensiones de este estudio, ya que los momentos que se tienen planeados (trabajo individual de la resolución del problema, reuniones en grupo sobre los avances y estrategias del problema y análisis metacognitivo) se vinculan y ajustan a ellas.

En relación con lo anterior, la conciencia de pensar y reflexionar antes, durante y después de cualquier situación problema, es lo que se determina como una acción metacognitiva, siendo la reflexión la acción clave que hace que el resolutor detenga por unos momentos el proceso de resolución y lo sitúe en un proceso metacognitivo consciente, con el objetivo de analizar y comprender las acciones cognitivas en relación con la resolución. Al respecto Ríos (citado en Jiménez, 2004) menciona que la metacognición comprende estas acciones como momentos del pensamiento reflexivo y presenta los indicadores de dichos componentes, entendiendo éstos como las acciones metacognitivas que se hacen a fin de lograr un objetivo cognitivo, las cuales se presentan en primera instancia en forma de interrogantes, lo que lleva al resolutor a indagar sobre determinados actos y razonamientos generados en la resolución, buscando dar sentido, orientación y trayectoria a los objetivos propuestos. 


\section{Metodología}

El estudio se ubica en un enfoque cualitativo, ya que se pretende describir y analizar la realidad tal y como sucede a partir del proceso llevado por los investigadores en la resolución de problemas, y de esta manera, poder interpretar y dar sentido a la información obtenida. Para ello se crean 3 fase que permiten la identificación, recolección y posterior análisis de la información, como primera se encuentra la fase epistemológica, definiéndose en ella el objeto de estudio para esta investigación, el cual es los procesos metacognitivos que se ubican en la resolución de un problema. Para la siguiente fase, la de estrategia general, se definen cuatro momentos que se darán de manera cíclica en la resolución de los dos resolutores, el primero es la resolución individual, seguido de la reunión de los resolutores, la recolección de la información y análisis de la información. En el primer momento, la resolución individual, cada uno de los resolutores realiza de manera individual la resolución del problema, plasmando en el cuaderno resolutor los distintos procesos generados y capturando en grabaciones de audio y video todos los razonamientos que el resolutor devela en tal proceso, para ello éste intentará revelar los distintos pensamientos y razonamientos de manera oral, lo cual se toma como "pensar el voz alta", a fin de permitir la mayor recolección de información posible, además, cada resolutor deberá realizar una descripción de su proceso de resolución, la cual es tomada como una primera recolección de información. En el siguiente momento, la reunión de los resolutores se presenta cada uno de los procesos realizados por los dos resolutores, se analizan los aspectos comunes y se centra la atención en los procesos que se consideren más relevantes (en pro de la resolución) o que generan mayor dificultad; para recoger la información de las socializaciones, se hace un acta donde se registran las discusiones y conclusiones obtenidas. El siguiente momento, el de recolección de la información, los resolutores toman el papel de investigadores identificando las distintas acciones metacognitivas que se pueden evidenciar en las grabaciones de audio y video de los procesos de resolución. Por último se encuentra el cuarto momento, el de análisis de la información en donde los investigadores tendrán como objetivo caracterizar, definir y distinguir cada una de las acciones metacognitivas identificadas, así como analizar la implicación de cada una de las acciones en la resolución de problemas.

Luego de contar con las herramientas conceptuales y tener claridad en los aspectos metodológicos, se crean tres categorías para llevar a cabo el análisis de las acciones metacognitivas, la primera fase, planificación/ abordaje; en la cual se planea el curso de la acción cognitiva, organiza y selecciona las estrategias que llevan a alcanzar el objetivo, supervisión/ ataque; donde se reflexiona sobre las operaciones mentales que están en marcha y examina sus consecuencias, evaluación/supervisión; donde se regulan y valoran los objetos que se han propuesto en el proceso de planificación y supervisión.

Acciones planificación: Interioriza y define el problema, reconociendo a dónde quiere llegar; reflexiona reglas y condiciones; delibera frente a qué se debería hacer primero; se cuestiona por cuáles acciones ayudarían en la tarea; busca representaciones y formas de organizar la información mediante símbolos, diagramas, tablas o gráficos; intenta anticipar las consecuencias de las acciones y define un plan de acción. Acciones supervisión: recapacita frente a como se ha hecho, examina acciones que se debería recordar, indaga que estrategias ha usado y de que otra manera se podría haber hecho, se cuestiona sobre la efectividad de las estrategias de solución, revisa sus razonamientos para encontrar posibles errores, deconstruye ideas cuando no se entiende algo, reorienta sus acciones con base en los posibles errores encontrados. Acciones revisión: recapacita frente a como se realizó el proceso, se cuestiona frente a que se podría haber hecho de otra forma, reconoce que tipo de razonamiento ha utilizado, delibera como se puede aplicar el razonamiento a otros problemas, autorregula los objetivos propuestos y los resultados alcanzados, reflexiona sobre la mejor solución, inspecciona la validez y pertinencia de las estrategias aplicadas.

\section{Análisis de los resultados}

En este apartado se presenta parte del análisis metacognitivo hecho a los procesos de resolución realizados por los dos resolutores, exponiendo un solo ejemplo debido a la complejidad, luego que cada uno de éstos se construyen a partir de una 
contextualización corta con el momento de la resolución en la que se ubica el resolutor, explicación del por qué se considera una acción metacognitiva, relación con la acción metacognitiva a la que hace referencia y definición dentro de la categoría metacognitiva en la que se clasifica.

Resolutor 1: A ver, como interpretamos esto, esos tres puntos deben estar unidos por funciones digo yo, pero me faltaría una función ahí, porque solo veo dos funciones, entonces me faltaría una, me falta una función, ¿Qué función me falta?... estoy confundidisimo porque solamente veo dos funciones, vamos a cambiar de lugar geométrico. Ahora me confundí porque estoy haciendo la construcción y me falta una, estoy haciendo la transformación del triángulo y pues yo sé que los tres puntos deben estar unidos por funciones, por funciones cuadráticas porque son tres puntos, es como coger tres segmentos pero no encuentro si no dos, entonces voy a hacer de nuevo la construcción a ver qué pasó.

En las observaciones que intenta hacer el resolutor, se pueden identificar dos acciones que se desarrollan al tiempo. Una se da en el momento en el que el resolutor se indaga por resultados que había previsto y la segunda cuando resalta su estado de confusión frente a dicha situación prevista. La primera es una acción metacognitiva debido a que el resolutor en medio del camino contrasta lo pensado con lo encontrado (acción autorregular), que lo lleva a sentirse confundido y por ende atascado, y por lo cual se produce la segunda acción; que es metacognitiva gracias a que el sentimiento de confusión lo hace revisar cada uno de sus razonamientos a fin de identificar errores y/o reconstruir el camino (acción Deconstruye) para salir del atasco. En este sentido, se puede apreciar que las dos acciones se dan de manera cíclica, aunque pertenezcan a categorías diferentes; los procesos de autorregulación se relacionan como acción de la categoría de revisión, puesto que el resolutor establece un paralelo entre los objetivos propuestos y los resultados encontrados en la marcha, y por otro lado, la reflexión constante del paralelo planteado entre la reorganización de los razonamientos en marcha y los que se generan producto de la revisión, se relaciona con la categoría de supervisión dado que el resolutor revisa y recrea los razonamientos a fin de esclarecer el camino y salir de su estado de estancamiento.

\section{Conclusiones}

Gracias al proceso de análisis de la resolución de los dos resolutores se concluye que si es posible hacer evidentes ciertas acciones metacognitivas relacionadas con la toma de conciencia, las cuales no se dan de manera lineal sino de manera cíclica dependiendo de los objetivos del resolutor. Además es de resaltar que es posible que en la resolución se produzcan acciones metacognitivas que se dan de manera simultánea y que, dependiendo de la relación de las mismas se puede generar un avance en la resolución.

Se encuentra similitud entre las fases de la resolución de problemas mencionadas por Mason, Burton y Stacey (1989) abordaje, ataque y revisión y las de Rios (citado en Jimenez, 2004) referentes a las dimensiones metacognitivas; planificación, supervisión y evaluación, dado que las distintas acciones cognitivas y metacognitivas mantienen regularidad en cuanto a los procesos que se dan en cada una y más específicamente guardan concordancia entre los objetivos para el desarrollo de la resolución.

El estudio queda abierto al análisis de la relación entre las acciones metacognitivas y la identificación de alguna de otra índole, es decir, la toma de conciencia plasmada en un gráfico, una actitud o cualquier otra acción que dé cuenta de un proceso metacognitivo en resolución de problemas matemáticos que no muestre de manera verbal.

\section{Referencias}

Jiménez, V (2004) Metacognición y comprensión de la lectura: evaluación de los componentes estratégicos (procesos y variables) mediante la elaboración de una escala de conciencia lectora (Escola) (Tesis Doctoral). Universidad Complutense de Madrid, Madrid, España.

Mason, Burton y Stacey (1989) Pensar matemáticamente. Madrid, España: Editorial Labor. 